\title{
Formation and Evolution of Pulsars \& Accretion and Jets of Black Holes
}

\author{
Cuixiang Zhong \\ Department of Physics, Jiangxi Normal University, Nanchang, China
}

Email address:

cuixiang_zhong@163.com

\section{To cite this article:}

Cuixiang Zhong. Formation and Evolution of Pulsars \& Accretion and Jets of Black Holes. American Journal of Astronomy and Astrophysics. Vol. 6, No. 3, 2018, pp. 91-96. doi: 10.11648/j.ajaa.20180603.15

Received: October 11, 2018; Accepted: November 2, 2018; Published: November 29, 2018

\begin{abstract}
The discovery of pulsar is an exciting discovery in 1960s, it has a profound influence on the development of modern physics. Although after the discovery of the first pulsar, it is quickly confirmed that pulsars are rapidly rotating neutron stars, yet people knew little about the essential mechanism leading neutron stars to pulse electromagnetic radiation. Thus, the author has analyzed the morphology and atmospheric environment of neutron stars, and found that a neutron star usually has two vortex structures located at its South pole and North pole, consisting of two groups of parallel spiral cloud paths, therefore producing two groups of corresponding dipole magnetic fields located at the South pole and the North pole respectively. It is the superposition of these two groups of dipole magnetic fields with the same polarity that form the neutron star's magnetic field continuously giving off radio and X-ray pulsations in lighthouse-like beams. Since the atmospheric vortexes on the planets of the Solar System are tiny accretion disks, and the accretion disks on neutron stars, black holes or active galactic nuclei are essentially large-scale atmospheric vortexes, neutron star's vortex-formation mechanism and electromagnetic radiation mechanism can be extended to the accretion and jets of black holes.
\end{abstract}

Keywords: Pulsars General, Neutron Stars, Radiation Mechanisms General, Accretion, Jets, Black Holes

\section{Introduction}

Pulsar is one kind of the most mysterious celestial bodies in the universe. Its high density, temperature, pressure and strength of magnetic field are beyond the imagination of people, therefore arousing the great interest and curiosity of people. Jocelyn Bell Burnell and Antony Hewish observed the first pulsar on November 28, 1967 [1, 2], then it is quickly confirmed that pulsars are rapidly rotating neutron stars, making the neutron star turn from hypothesis to fact [3]. This is really a great celebration of astronomy in the last century. Hence, the discovery of pulsar is known as one of the four major astronomical discoveries in 1960s, and also Antony Hewish and Martin Ryle became the first astronomers to be awarded the Nobel Prize in physics in 1974. Since the first pulsar has been discovered, no less than 1620 pulsars have been found, and they are all found in the Milky way [4, 5]. One notable feature of pulsar is its stable and short pulse period [6]. How does such a kind of regular pulse come into being? This problem is still in pending. Fortunately, the author has restudied the formation and evolution of pulsars, and found a reasonable answer.

\section{Existing Theory of the Formation of Pulsar}

The discovery of pulsar and its singularity have prompted people to make a series of research on the formation and evolution of pulsar. The preliminary conclusion drawn by astronomers is that the formation of a pulsar begins when the core of a massive star is compressed during a supernova, which collapses into a neutron star. The neutron star retains most of its angular momentum, and since it has only a tiny fraction of its progenitor's radius (and therefore its moment of inertia is sharply reduced), it is formed with very high rotation speed. Meanwhile, the compression makes the neutron star a strong magnet. A beam of radiation is emitted along the magnetic axis of the pulsar, which spins along with the rotation of the neutron star. The magnetic axis of the pulsar determines the direction of the electromagnetic beam, with the magnetic axis not necessarily being the same as its rotational 
axis. This misalignment causes the beam to be seen once for every rotation of the neutron star, which leads to the "pulsed" nature of its appearance.

In rotation-powered pulsars, the beam originates from the rotational energy of the neutron star, which generates an electrical field from the movement of the very strong magnetic field, resulting in the acceleration of protons and electrons on the star surface and the creation of an electromagnetic beam emanating from the poles of the magnetic field. This rotation slows down over time as electromagnetic power is emitted [7] When a pulsar's spin period slows down sufficiently, the radio pulsar mechanism is believed to turn off (the so-called "death line"). This turn-off seems to take place after about 10-100 million years, which means of all the neutron stars born in the 13.6 billion year age of the universe, around $99 \%$ no longer pulsate [6].

Though the general picture of pulsars as rapidly rotating neutron stars is widely accepted, Werner Becker of the Max Planck Institute for Extraterrestrial Physics said in 2006, "The theory of how pulsars emit their radiation is still in its infancy, even after nearly forty years of work." [8]

\section{A New Theory of the Formation of Pulsar}

Although the lighthouse model has been accepted by most experts, it still has several difficult questions to answer: why can a star become a magnet when it is compressed into a neutron star? When a magnet rotates at a high speed, can it really emit an electromagnetic beam from the magnetic axis? All these questions make the above lighthouse model incredible. In fact, the lighthouse model is simply generalized from the hypothesis of geomagnetic field formation. However, Earth and neutron star have different structures, that is, Earth's outer core is liquid while neutron stars are the densest stars, so the above generalization is wrong. Besides, the reason for the formation of the geomagnetic field is still not clear, so it is lack of solid scientific foundation to generalize the hypothesis of geomagnetic field formation to the hypothesis of pulsar magnetic field formation.

When a star's magnetic field is studied, not only the inner structure of the star should be considered but also the outer environment of the star should be paid more attention. For example, the well-known Earth has a dense atmosphere. Due to the effect of centrifugal force, the rotation of Earth around its axis has caused it to bulge around the Equator, making the Earth become an oblate spheroid with the radius of the two poles of the Earth is less than the radius of the equator and other places, while the gravitational force is inversely proportional to the square of the distance. When the Earth rotates quickly, the rotation will produce strong centrifugal force, making the clouds over the equator and low latitudes tend to move away from their orbits to the South Pole or the North Pole. Because the gravitational attraction of the polar position is greater than that of other locations, when clouds move above the polar regions, they are easily attracted by the gravitational pull of the polar regions, after inhaling cold air, they condense into thick clouds and sink gradually. Many polar-plunging clouds form a strong circulation around the pole as the Earth rotates, that is polar vortex, as is shown in Figure 1. The Earth has two vortexes, located at the South Pole and the North Pole respectively, which can span troposphere and stratosphere. This kind of vortex structures exists throughout the four seasons, reaching maximum strength in winter. There are also vortexes on Venus, Mars, Jupiter and Saturn of the Solar System. The polar vortex of Saturn is particularly spectacular, as is shown in Figure 2, which is captured by NASA's Cassini spacecraft. It is a persisting hexagonal cloud pattern around the north pole of Saturn. The sides of the hexagon are about 13,800 km long, which is more than the diameter of Earth (about 12,700 km). The spiral current produced by Jupiter's arctic vortex is also spectacular, as is shown in Figure 3, which is captured by NASA's Hubble Space Telescope.

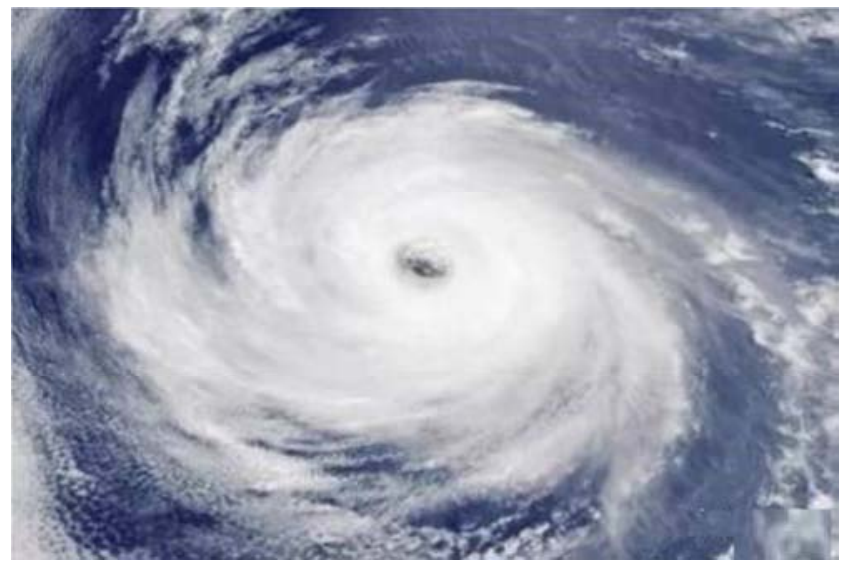

Figure 1. Arctic polar vortex of Earth.

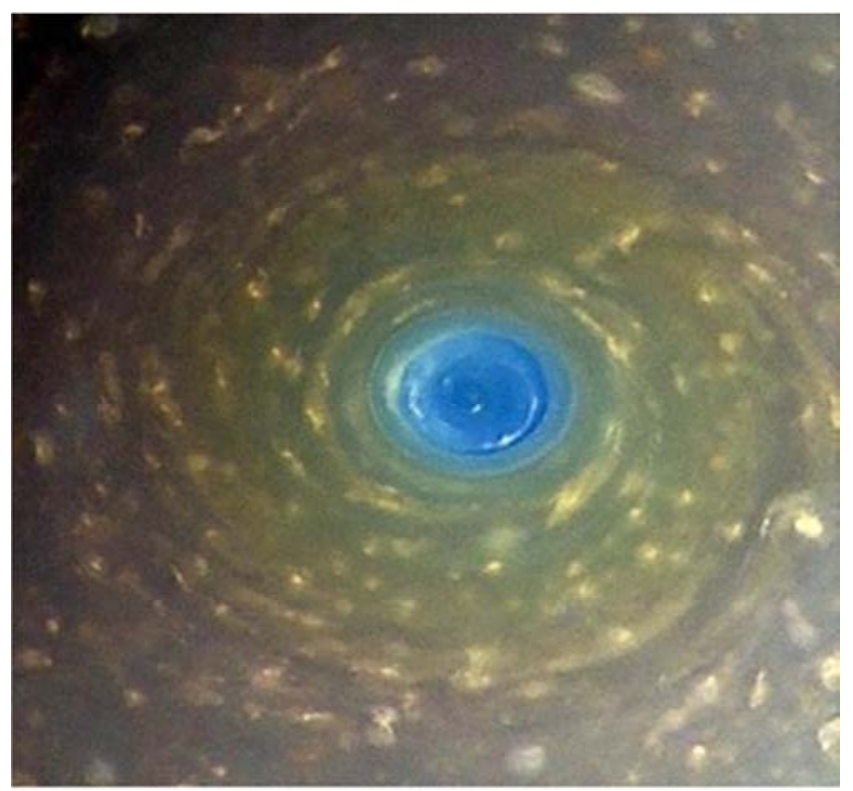

Figure 2. The polar vortex of Saturn. 


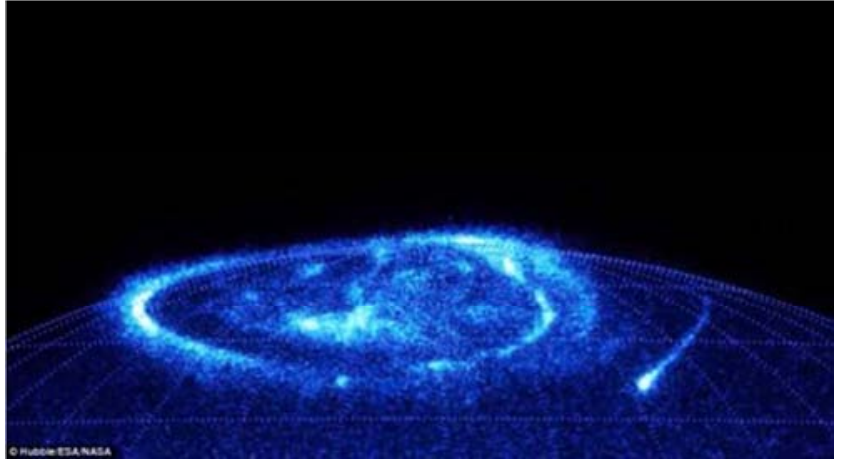

Figure 3. The spiral current produced by Jupiter's arctic vortex

It's well known that a pulsar is a rapidly rotating neutron star, it is the product of a supernova explosion. Due to the effect of centrifugal force, the rotation of a neutron star around its axis has caused it to bulge around the Equator, making the neutron star become an oblate spheroid with the radius of the two poles is less than the radius of the equator and other places, as is shown in Figure 4. Although the neutron star is small, its mass is much larger than that of the Sun, not to mention that of Earth. Hence, the nebula around a neutron star is much thicker and denser than the Earth's atmosphere, respectively, this is the reason why the neutron star is hard to observe[9]. When a neutron star rotates quickly, the rotation will produce strong centrifugal force, making the clouds over the equator and low latitudes tend to move away from their orbits to the South Pole or the North Pole. Because the gravitational attraction of the polar position is greater than that of other locations, when clouds move above the polar regions, they are easily attracted by the gravitational pull of the polar regions, after inhaling cold air, they condense into thick clouds and sink gradually. Many polar-plunging clouds form a strong circulation around the pole as the neutron star rotates, that is polar vortex of neutron star. Since the clouds involved in polar vortex are numerous and continuous, and revolve rapidly, it is easy to have violent frictions and collisions among clouds, which can generate violent and continuous thunder and lightning, as is shown by Figure 5 .

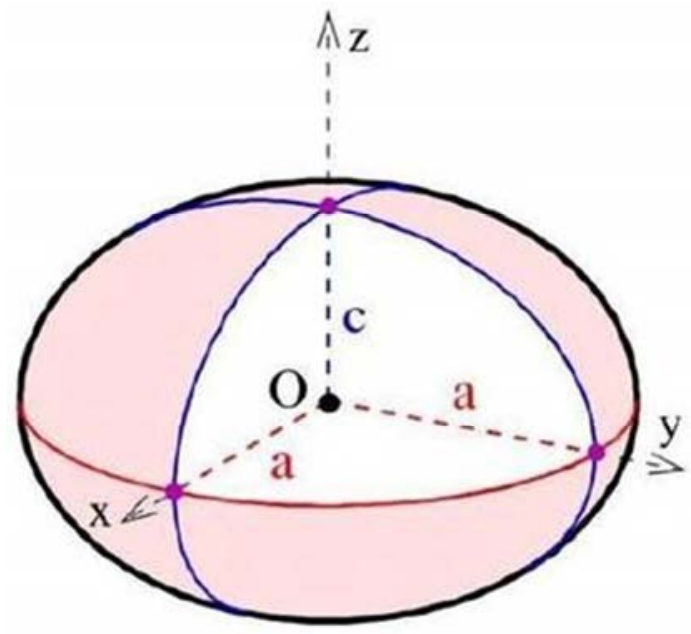

Figure 4. A neutron star or black hole is an oblate spheroid.

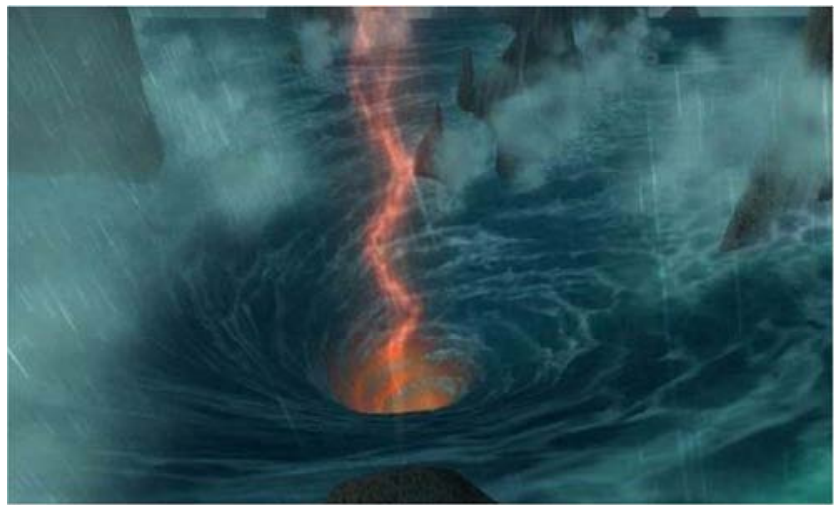

Figure 5. Continuous radio from the polar vortex of neutron star.

Since the clouds involved in a polar vortex are constantly in flux and revolve downward rapidly in a spiral manner, a series of parallel thick spiral cloud paths can be formed, which facilitate the downward flow of heavier negatively charged cloud droplets, thus forming a series of good circuits with excellent electrical conductivity. Since the clouds involved in polar vortex are numerous and revolve rapidly, it is easy to have violent frictions and collisions among clouds, making the vortexes filled with positive ions and negative ions. Cloud droplets must first absorb negative ions in the atmosphere, causing the droplets to be negatively charged, and the larger cloud droplets fall toward the lower part of the cloud or even the lower portion of the vortex along a spiral cloud path, while the lighter positive ions are gradually brought up by the updraft to the upper part of the cloud or even the upper portion of the vortex along the spiral cloud path, forming a current from the lower portion of the vortex to the upper portion of the vortex along the spiral cloud path. In addition, since the clouds along a spiral cloud path are numerous and revolve rapidly, it is easy to have violent frictions and collisions among clouds, producing frequent electrical discharge or thunderstorms. Each electrical discharge or thunderstorm acts as an electrostatic motor, which can send currents to the upper portion or the lower portion of the vortex, preponderantly forming an intense current following the cloud path from the bottom of the vortex to the top of the vortex. Since there is a continuous flow of currents along the spiral cloud path from the bottom of the vortex to the top of the vortex, thus forming a powerful dipole magnetic field with its magnetic North Pole pointing towards the South Pole of the neutron star and its magnetic South Pole pointing towards the North Pole of the neutron star, as is shown in Figure 6. Since the neutron star has two vortex structures located at the South Pole and the North Pole, consisting of two groups of parallel spiral cloud paths, therefore it has two groups of corresponding dipole magnetic fields located at the South pole and the North Pole respectively. It is the superposition of these two groups of dipole magnetic fields of the same polarity that form the present magnetic field of the neutron star. This magnetic field continuously gives off radio and X-ray pulsations in lighthouse-like beams. During the rotation of a neutron star, whenever such a vortex is pointing to the Earth, people on 
Earth can receive electromagnetic pulses from the pulsar, as is shown in Figure 7.

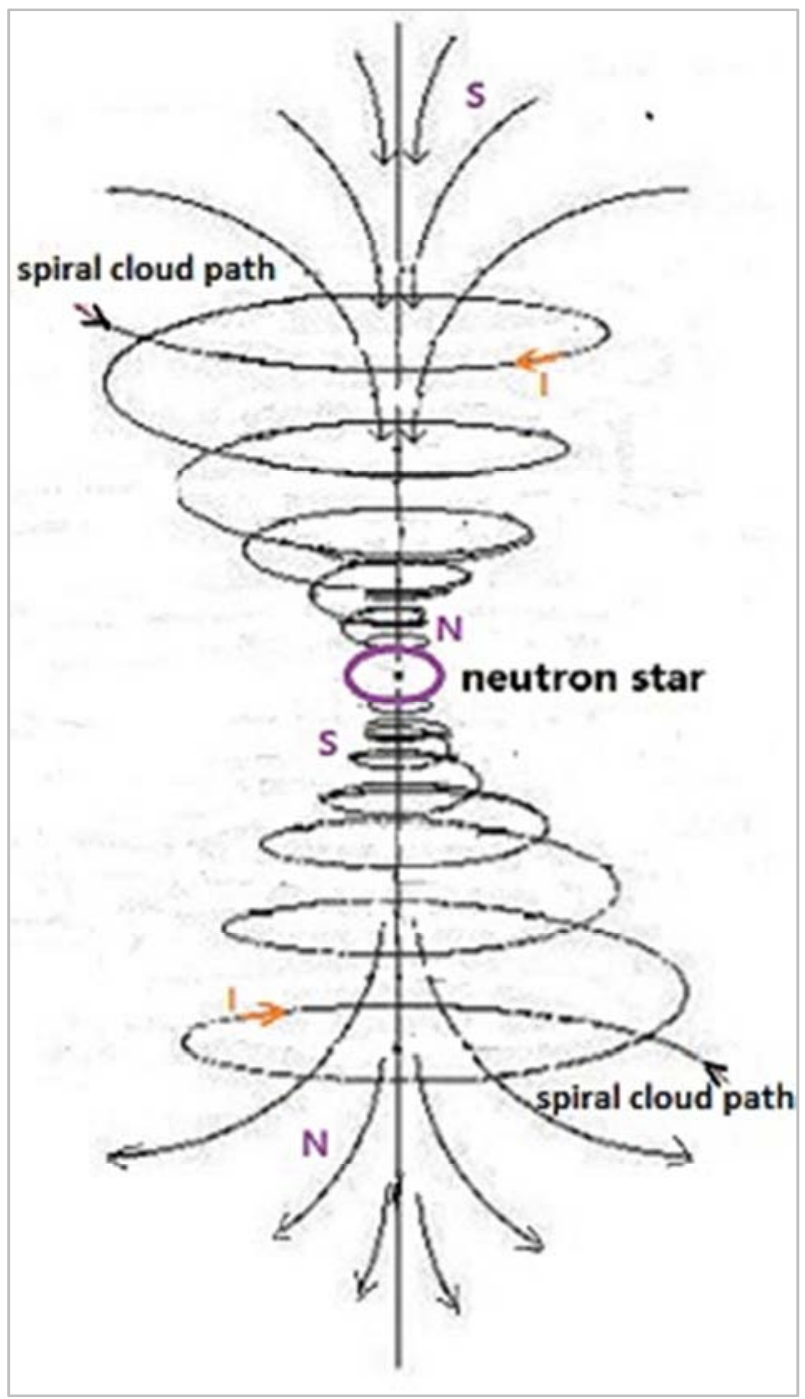

Figure 6. A neutron star emitting electromagnetic beam.

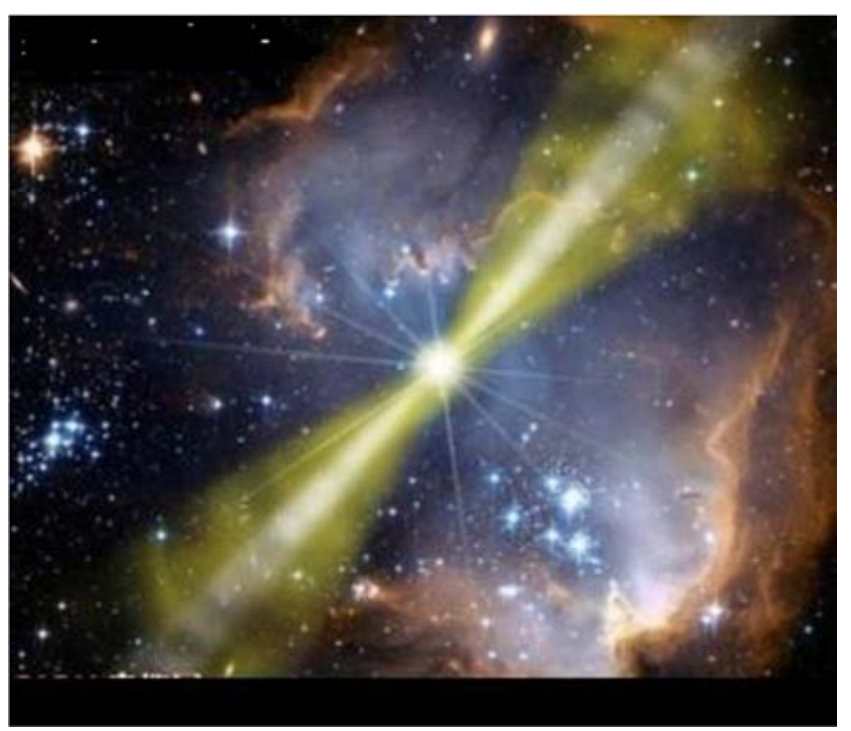

Figure 7. A pulsar emitting electromagnetic pulses.

\section{The Evolution of Pulsar}

It's well known that a pulsar is a rapidly rotating neutron star, it is the product of a supernova explosion, but it is not static. During the movement of a galaxy, a neutron star unceasingly incorporates the nebula materials near the orbits to increase its own mass and volume as well as thicken its own atmosphere, but its rotation speed will decrease gradually. When the atmosphere of the star becomes very dense and its surface is covered with a thick layer of material again, the conditions of a thermonuclear reaction are satisfied again, thus a supernova explosion occur again. But such a supernova explosion will soon consume the accumulated atmosphere and surface material, making the neutron star collapse again and the stars shrink greatly, obtaining a more massive rapidly rotating neutron star. Thus it can be seen that during the evolution of a pulsar into a massive star, supernova explosion may occur multiple times, each time produces a more massive neutron star[10-13]. When the atmosphere of the neutron star is thickened again, a new pulsar is ready. With the increase of the neutron star's mass, it will finally become a black hole.

\section{From the Formation and Evolution of Pulsars to the Accretion and Jets of Black Holes}

According to the previous discussion, we know that there are vortexes on Venus, Earth, Mars, Jupiter and Saturn of the Solar System. Although the vortexes of Earth and Saturn can span troposphere and stratosphere, yet looking from the images taken by astronomical telescopes or satellites, they all resemble accretion disks on massive stars, as is shown in Figure 1 and Figure 2. On the other hand, some international research teams have observed the whole process of a supermassive black hole gobbling up a star flung into reach of its enormous gravity, this result has verified the existing black hole accretion disk theory: an accretion disk is a disk structure formed by the material falling into a central celestial body due to gravitation. Gravitation causes orbiting material in the disk to spiral inward towards the central celestial body. Gravitational and frictional forces compress the material and raise the temperature of the material, causing the emission of electromagnetic radiation. Thus, it can be deduced that the atmospheric vortexes on the above planets are tiny accretion disks, and the accretion disks on neutron stars, black holes or active galactic nuclei are large-scale atmospheric vortexes[14-17]. Hence, neutron star's vortex-formation mechanism and electromagnetic radiation mechanism can be extended to the accretion and jet of black holes .

Although the existing black hole accretion disk theory can basically explain the formation process of black

hole accretion disk, yet it can not reveal the true structure and geographical location of accretion disk. This theory, only based on the observed images, concluded that a black hole accretion disk is a disk structure, not knowing the true structure of black hole accretion disk is a large-scale vortex 
structure. This theory also mistakenly believes that accretion disk surrounds the central celestial body and rotates around it, not knowing the accretion disk is a vortex structure formed in the star polar regions and rotating around the axis of star rotation. In fact, there is a pair of large-scale vortices in the two polar regions of the star, as is shown in Figure 8 . In addition, the existing black hole accretion disk theory fails to solve the following problem: Why do accretion disks emit jets along their polar axes? [18-20]

During the evolution of a neutron star into a massive star, supernova explosions may occur multiple times, each supernova explosion can produce a neutron star with greater mass and faster rotation, eventually the neutron star becomes a high speed rotating black hole. Due to the effect of centrifugal force, the rotation of a black hole around its axis has caused it to bulge around the Equator, making the black hole become an oblate spheroid with the radius of the two poles is less than the radius of the equator and other places, as is shown in Figure 4. Since a black hole's mass is much larger than that of Earth, the nebula around the black hole is much thicker and denser than the Earth's atmosphere, this is the reason why the black hole is hard to observe. When a black hole rotates quickly, the rotation will produce strong centrifugal force, making the clouds over the equator and low latitudes tend to move away from their orbits to the South Pole or the North Pole. Because the gravitational attraction of the polar position is greater than that of other locations, when clouds move above the polar regions, they are easily attracted by the gravitational pull of the polar regions, after inhaling cold air, they condense into thick clouds and sink gradually. Many polar-plunging clouds form a strong circulation around the pole as the black hole rotates, that is polar vortex of black hole. Since the clouds involved in polar vortex are numerous and continuous, and revolve rapidly, it is easy to have violent frictions and collisions among clouds, especially at the bottom of the vortex, which can generate violent and continuous lightning and jets. Because the shape of the atmospheric vortex is like a conical funnel, and there is a long cylinder at the bottom of this funnel, the jets generated from this cylinder will shoot along the central axis of the cone or polar axis, forming a cosmic fire, as is shown in Figure 8.

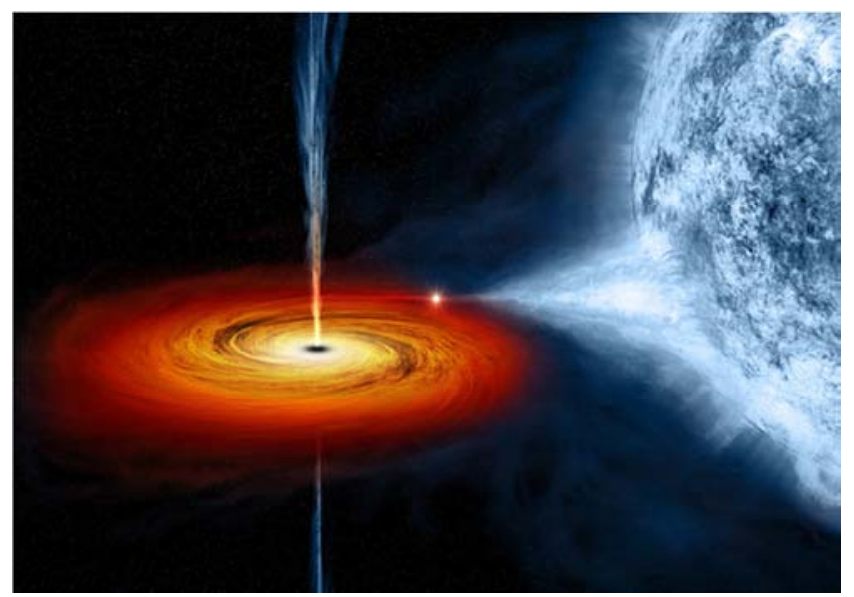

Figure 8. Jets of black hole.

\section{Conclusion and Prospect}

The discovery of pulsar is one of the four great discoveries of astronomy in 1960s. It opens up a new way for mankind to explore nature, and has a profound influence on the development of modern physics. Although after the discovery of the first pulsar, it is quickly confirmed that pulsars are rapidly rotating neutron stars, yet the problem why neutron stars emit beams of electromagnetic radiation has suspended for half a century. Fortunately, the author has analyzed the morphology and atmospheric environment of neutron stars, and found the essential mechanism, which can be used to solve all the problems relating to the characters and features of neutron stars. Hence, it a major leap in pulsar research. In addition, since the accretion disks on neutron stars, black holes or active galactic nuclei are all large-scale atmospheric vortexes, neutron star's vortex-formation mechanism and electromagnetic radiation mechanism can be extended to the accretion and jet of black holes.

\section{References}

[1] Bell Burnell, S. Jocelyn. Little Green Men, White Dwarfs, or Pulsars? Annals of the New York Academy of Science, vol. 302, pages 685-689, Dec., 1977.

[2] Bell Burnell, S. Jocelyn (23 April 2004). So Few Pulsars, So Few Females . Science. 304 (5670): 489.

[3] Gold, T. (1968). "Rotating Neutron Stars as the Origin of the Pulsating Radio Sources". Nature. 218 (5143): 731.

[4] Deneva, J. S.; Cordes, J. M.; Lazio, T. J. W. (2009). Discovery of Three Pulsars from a Galactic Center Pulsar Population. The Astrophysical Journal Letters. 702 (2): L177-182.

[5] D. Backer; Kulkarni, Shrinivas R.; Heiles, Carl; Davis, M. M.; Goss, W. M. (1982). A millisecond pulsar. Nature. 300 (5893): 315-318.

[6] Matsakis, D. N.; Taylor, J. H.; Eubanks, T. M. (1997). A Statistic for Describing Pulsar and Clock Stabilities. Astronomy and Astrophysics. 326: 924-928.

[7] Pacini, F. (1967). Energy Emission from a Neutron Star. Nature. 216 (5115): 567.

[8] Young, M. D.; Manchester, R. N.; Johnston, S. (1999). A Radio Pulsar with an 8.5 -Second Period that Challenges Emission Models. Nature. 400 (6747): 848-849.

[9] Lyne, Andrew G.; Graham-Smith, Francis. Pulsar Astronomy. Cambridge University Press, 1998.

[10] Arcavi, Iair; et al. (2017). Energetic eruptions leading to a peculiar hydrogen-rich explosion of a massive star. Nature. 551 (7679): 210

[11] Cho, A. (16 February 2018). A weight limit emerges for neutron stars. Science. 359 (6377): 724-725.

[12] Margalit, B.; Metzger, B. D. (2017-12-01). Constraining the Maximum Mass of Neutron Stars from Multi-messenger Observations of GW170817. The Astrophysical Journal. 850 (2): L19. 
[13] Ruiz, M.; Shapiro, S. L.; Tsokaros, A. (2018-01-11). GW170817, general relativistic magnetohydrodynamic simulations, and the neutron star maximum mass. Physical Review D. 97 (2): 021501.

[14] Gurzadyan, V. G.; Ozernoy, L. M. (1979). Accretion on massive black holes in galactic nuclei. Nature. 280.

[15] Floyd, David J. E.; Bate, N. F.; Webster, R. L. (2009). The accretion disc in the quasar SDSS J0924+0219. Monthly Notices of the Royal Astronomical Society. 398 (1): 233239.

[16] Beckwith, K.; Hawley, J. F.; Krolik, J. H. (2009). Transport of large-scale poloidal flux in black hole accretion. Astrophysical Journal. 707 (1): 428-445.
[17] Poindexter, Shawn; Morgan, Nicholas; Kochanek, Christopher S. (2008). The Spatial Structure of An Accretion Disk. The Astrophysical Journal. 673 (1): 34-38.

[18] Beckwith, K.; Hawley, J. F.; Krolik, J. H. (2009). TRANSPORT OF LARGE-SCALE POLOIDAL FLUX IN BLACK HOLE ACCRETION (PDF). Astrophysical Journal. 707 (1): 428-445.

[19] Daniel Clery. (2014). What powers a black hole's mighty jets? Science $\mid A A A S$. Nov. 19, 2014.

[20] Johnson, M. D.; Fish, V. L.; Doeleman, S. S.; Marrone, D. P.; Plambeck, R. L.; Wardle, J. F. C.; Akiyama, K.; Asada, K.; Beaudoin, C. (4 December 2015). Resolved magnetic-field structure and variability near the event horizon of Sagittarius A*. Science. 350 (6265): 1242-1245. 